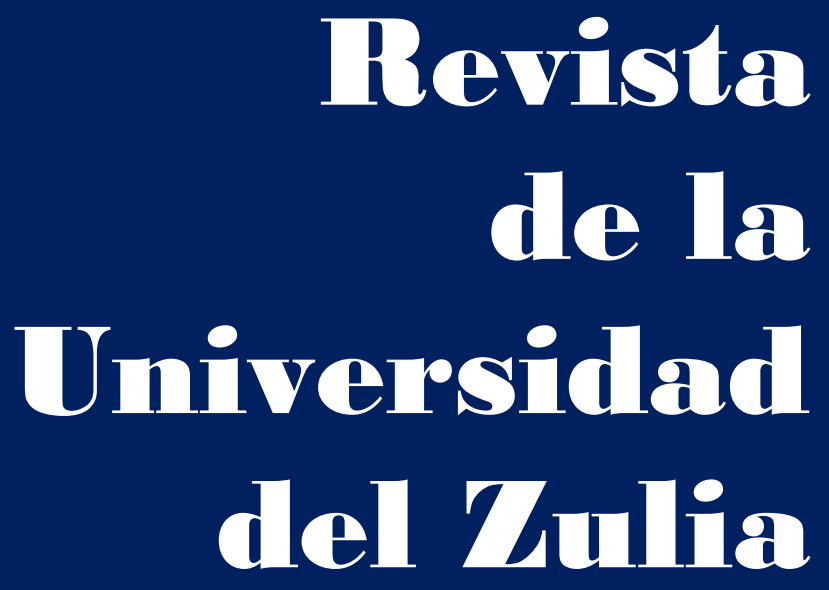

Fundada en 1947

por el Dr. Jesús Enrique Lossada

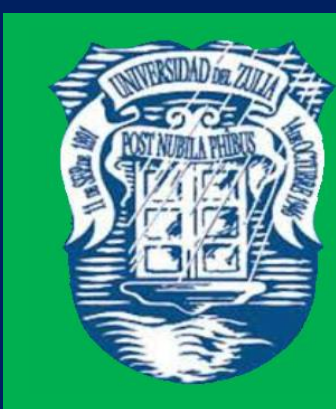

Ciencias del

Agred

Ingemieria

y Teenología
Añต 12 No 32

Enero - Abril 2021

Tercera Época

Maracailbo-Venezuela 
REVISTA DE LA UNIVERSIDAD DEL ZULIA. 3época. Año $12 \mathrm{~N}^{\circ}$ 32, 2021

Eder Nicanor Figueroa Piscoya et al. // Modelo de calidad para plataformas e-learning ... 330-343

DOI: http://dx.doi.org/10.46925//rdluz.32.20

\title{
Modelo de calidad para plataformas e-learning basado en normas ISO/IEC 25000
}

\author{
Eder Nicanor Figueroa Piscoya* \\ Gilberto Carrión-Barco** \\ Manuel-Jesús Sánchez-Chero *** \\ Andrés Heleodoro Figueroa Alvarado**** \\ Walter Antonio Figueroa Neyra*****
}

RESUMEN

La presente investigación tuvo por objetivo desarrollar un modelo de calidad para plataformas e-learning en centros de entrenamiento superior, basado en estándares ISO/IEC 25000; se utilizó el enfoque cuantitativo con alcance propositivo y con diseño no experimental de tipo transversal; se empleó el cuestionario como instrumento de recolección de datos conformado por ll reactivos y se aplicó a una muestra de 20 sujetos entre personal administrativo y docente del Centro de Tecnologías de la Información CETI - Chiclayo-Perú. Los resultados indican que la plataforma Blackboard y la plataforma Moodle son las preferidas en la implementación de entornos virtuales de aprendizaje; por su parte El $80 \%$ señala que el seguimiento de los avances del estudiante en tiempo real es la característica más importante que incorporarían en la implementación de una plataforma e-learning. Por ello, se propuso un modelo de calidad para plataformas e-learning en centros de entrenamiento superior, basado en estándares ISO/IEC 25000. Como conclusión se precisa que la adopción de una plataforma e-learning en los centros de entrenamiento superior es imprescindible por lo que se hace necesario desarrollar un modelo de calidad para estas plataformas.

PALABRAS CLAVE: Plataformas, e-learning, ISO/IEC 25000, modelo.

*Docente Auxiliar: Universidad Nacional Toribio Rodríguez de Mendoza. Chachapoyas. Perú. ORCID: https://orcid.org/0000-0001-7970-434X. E-mail: eder.figueroa@untrm.edu.pe

**Docente Auxiliar. Universidad Nacional Pedro Ruiz Gallo. Perú. ORCID: https://orcid.org/0000-00021104-6229. E-mail: gcarrion@unprg.edu.pe

***Docente Investigador. Universidad Nacional de Frontera. Perú. ORCID: https://orcid.org/0000-00031646-3037. E-mail: manuelsanchezchero@gmail.com

****Docente Principal. Universidad Nacional Pedro Ruiz Gallo. Perú. ORCID: https:/orcid.org/0000-00026135-2507. E-mail: afigueroa@unprg.edu.pe

*****Docente a Tiempo Completo. Universidad Peruana de Ciencias Aplicadas. Perú. ORCID: https://orcid.org/0000-0003-4747-9234. E-mail: pcmawfig@upc.edu.pe

Recibido: 15/10/2020

Aceptado: 10/12/2020 


\section{Quality model for E-learning platforms based on ISO/IEC 25000 standards}

ABSTRACT

The objective of this research was to develop a quality model for e-learning platforms in higher training centers, based on ISO / IEC 25000 standards; The quantitative approach was used with a propositional scope and a non-experimental cross-sectional design; The questionnaire was used as a data collection instrument made up of 11 items and was applied to a sample of 20 subjects among administrative and teaching staff of the Information Technology Center CETI Chiclayo-Peru. The results indicate that the Blackboard platform and the Moodle platform are the preferred ones in the implementation of virtual learning environments; For their part, $80 \%$ point out that monitoring student progress in real time is the most important feature that they would incorporate in the implementation of an e-learning platform. Therefore, a quality model for e-learning platforms in higher training centers was proposed, based on ISO / IEC 25000 standards. As a conclusion, it is specified that the adoption of an e-learning platform in higher training centers is essential for what is necessary to develop a quality model for these platforms. KEY WORDS: Platforms, e-learning, ISO / IEC 25000, model.

Introducción

El software es una herramienta de mucha utilidad que se encuentra presente en numerosas actividades de la educación, salud, industria, economía, transporte, seguridad, las artes, etc. (Vaca \& Jácome, 2018), cuyo mayor provecho es la mejora de procesos con el propósito de ofrecer eficiencia y satisfacción de necesidades empresariales. No obstante, hacer las correcciones a un software existente o desarrollar uno nuevo, en repetidas ocasiones no se implementa de la forma correcta (Callejas-Cuervo et al., 2017).

En los últimos años, el uso de Internet se ha ido incrementado (Blázquez Barba et al., 2018), conquistando un importante espacio en la vida diaria de las personas, dado que actualmente es utilizado en diversos contextos con múltiples propósitos, demostrando ser una herramienta muy potente que contribuye en la reducción de la brecha digital (Morales Ramírez et al., 2020). La supremacía del Internet reside en su universalidad; por tanto, es una necesidad y una cuestión estratégica que todos logren acceder a este (Sanchez, 2011). 
REVISTA DE LA UNIVERSIDAD DEL ZULIA. 3época. Año $12 \mathrm{~N}^{\circ}$ 32, 2021

Eder Nicanor Figueroa Piscoya et al. // Modelo de calidad para plataformas e-learning ... 330-343

DOI: http://dx.doi.org/10.46925//rdluz.32.20

En relación con otras ciencias, la computación como tal y el desarrollo de soft ware acarrea poco más de medio siglo en ser investigada y casi dos décadas que se vienen aplicando normas internacionales ISO con el propósito de brindar calidad al producto final (ISO 25000, 2020). No obstante, la gestión de proyectos conexas con las Tecnologías de la Información (TI) todavía se rehúsa a utilizar estos estándares, por diversas razones.

En el informe CHAOS de Standish Group, tal como lo señala James (20018), se muestra que los proyectos concluidos con éxito "Succesful" se encuentran alrededor del 33\%, mientras que los concluidos pero con menor sobrecosto y/o alcance y/o fuera del tiempo "Challenged" están en el orden del 48\%, y los proyectos cancelados antes de completarse "Failed" alcanzan el $19 \%$.

De acuerdo con el reporte Pulse of the Profession PMI (2017) fueron consultados 3,234 especialistas en gestión de proyectos, los cuales indicaron que las causas del infortunio de los proyectos, principalmente son: recursos limitados (22\%), apoyo insuficiente del sponsor (27\%), comunicación inadecuada/deficiente (30\%), cambios en los objetivos del proyecto $(36 \%)$, recolección inexacta de requerimientos (39\%), entre otros. De estos profesionales consultados, el 18\% labora en el rubro de tecnología de la información y comunicación (TIC); en consecuencia, se puede puntualizar que los expertos en proyectos de TIC presentan inconvenientes en culminar de manera satisfactoria los proyectos, lo cual supone que el producto final posea limitaciones en cuanto a calidad.

El uso del e-learning para enseñanza en centros de entrenamiento superior progresa rápidamente, para los centros que ya cuentan con esta plataforma les es imperativo evaluar el nivel de calidad que posee esta herramienta tecnológica. La investigación fue realizada tomando como referencia a un centro de capacitación y formación en cursos de TIC; estos centros de entrenamiento nacen como añadidura a la preparación profesional, con el propósito de obtener en el alumno una formación plena en tecnologías emergentes, extendiendo la capacitación del profesional en beneficio de la empresa y la sociedad.

La calidad de las plataformas e-learning presenta ciertas limitaciones, así como lo menciona Gómez (2015), donde indica que los instructores al usar estas plataformas encuentran graves deficiencias al momento de calificar las actividades de los participantes, de discernir sus 
REVISTA DE LA UNIVERSIDAD DEL ZULIA. 3época. Año $12 \mathrm{~N}^{\circ}$ 32, 2021

Eder Nicanor Figueroa Piscoya et al. // Modelo de calidad para plataformas e-learning ... 330-343

DOI: http://dx.doi.org/10.46925//rdluz.32.20

conductas on-line y de valorar el propio entorno y la aplicación de la plataforma; denotándose el sinsabor del usuario final con esta tecnología; tal y como expresa Deming (1989), calidad es una sucesión de controversias conducentes hacia la mejora continua para alcanzar la complacencia del usuario.

Calidad es definida de diversas formas, es un término muy subjetivo y estudiado por muchos autores. En el contexto de las TIC, puntualmente en el desarrollo de software, según Roger Pressman, la definición de calidad se vincula a la congruencia entre el rendimiento y los requisitos funcionales expresamente determinados con esquemas de desarrollo documentados, y con características sobrentendidas que se pretenden de un software al más alto nivel (citado por Callejas-Cuervo et al., 2017). Fundamentalmente estos requerimientos son la base del fracaso o éxito de los proyectos, ya que de ser gerenciados de forma equívoca podrían aterrizar pérdidas de tiempo y en sobrecostos.

Las organizaciones que evalúan sus productos de software utilizan estándares internacionales para la calidad, que son definidos para cuantificar el progreso en todo el ciclo de desarrollo hasta la puesta en funcionamiento del producto (Ramos et al., 2018). Desafortunadamente la cantidad de empresas que se rigen por estas normas es limitada, dado que al poner en marcha estos procesos para asegurar la calidad, conllevan un costo adicional y demandan un tiempo que en la mayoría de las veces las organizaciones no están dispuestas a invertir.

Las normas ISO/IEC 25000 se centran en la calidad del producto final de software y expresa los conceptos de calidad: de uso, externa e interna. La calidad del producto se ve mejorada por la eficiencia del proceso; a su vez, la calidad del producto colabora en mejorar la calidad en uso. Por consiguiente, evaluar y perfeccionar un proceso es una forma de optimizar la calidad del producto; evaluar y perfeccionar la calidad del producto es una forma de optimizar la calidad en uso. De igual manera, valuar la calidad en uso puede suministrar una retro alimentación para optimar el producto, y evaluando un producto puede facilitar un feedback para perfeccionar un proceso (Baldeón, 2015).

Entre los modelos para evaluar la calidad del producto se puede mencionar a McCall, siendo el primer modelo de medición de calidad de un producto de software; desde esta 
REVISTA DE LA UNIVERSIDAD DEL ZULIA. 3época. Año 12 N 32, 2021

Eder Nicanor Figueroa Piscoya et al. // Modelo de calidad para plataformas e-learning ... 330-343

DOI: http://dx.doi.org/10.46925//rdluz.32.20

perspectiva, la calidad estaba compuesta por tres criterios básicos: operación, revisión y transición; cada uno de estos criterios contenían, a su vez, una serie de métricas que determinaban la calidad del producto software (Miguel et al., 2014). El modelo propuesto por McCall sirvió como pilar para la creación de la primera norma estándar internacional orientada a la medición de la calidad del producto software en el año 1991: la ISO/IEC 9126, la cual está dividida en cuatro partes, cada uno con un propósito concreto: modelo de calidad, métricas internas, métricas externas y calidad de métricas en uso. Dentro de las métricas internas y externas la ISO/IEC 9126 precisa seis características; mientras que las métricas de calidad en uso identifican cuatro características (Miguel et al., 2014).

El presente estudio se realizó en el Centro de Entrenamiento en Tecnologías de la Información (CETI), ubicado en la Región Lambayeque, Provincia de Chiclayo - Perú. CETI es una empresa de capacitación dedicada a ofertar servicios de enseñanza y entrenamiento en diversas áreas de formación continua, dirigida a estudiantes, egresados, bachilleres, profesionales y empresas en el ámbito de las TIC. Los servicios de formación continua que ofrece CETI se enmarcan en el entrenamiento, capacitación, enseñanza y certificación de cursos relacionados a las TIC.

Ante esta situación problemática surge la siguiente interrogante; ¿De que manera se puede mejorar la calidad de las plataformas e-learning en los centros de entrenamiento superior?

Esta investigación se justifica porque en la actualidad aún existen entidades dedicadas al desarrollo de software que no siguen una tendencia de calidad por diversos factores, como desconocimiento de modelos de calidad, mala planificación de proyectos o carencia de modelos adaptados a exigencias específicas; obteniendo así software con calidad deficiente. Es allí donde los estándares ISO/IEC 25000 ofrecen una base esencial para la evaluación, modificación y creación de software, proporcionándoles la calidad adecuada; por su parte los centros de entrenamiento superior utilizan plataformas de aprendizaje sin tener en cuenta los requerimientos mínimos de calidad y las prestaciones del servicio educativo.

Ante lo expuesto se plantea la siguiente hipótesis: mediante el desarrollo de un modelo basado en normas ISO/IEC 25000 se garantizará la calidad de las plataformas e-learning como herramienta de aprendizaje en centros de entrenamiento superior. Por último, como objetivo de 
REVISTA DE LA UNIVERSIDAD DEL ZULIA. 3época. Año $12 \mathrm{~N}^{\circ}$ 32, 2021

Eder Nicanor Figueroa Piscoya et al. // Modelo de calidad para plataformas e-learning ... 330-343

DOI: http://dx.doi.org/10.46925//rdluz.32.20

la presente investigación se plantea: desarrollar un modelo de calidad para plataformas elearning en centros de entrenamiento superior basado en normas ISO/IEC 25000.

\section{Metodología}

El estudio investigativo tuvo un enfoque cuantitativo y alcance propositivo, con diseño no experimental de tipo transversal (Bernal, 2010); la muestra estuvo constituida por 20 sujetos entre docentes y administrativos de la institución CETI, teniendo como criterios de inclusión a los docentes y administrativos de la entidad, y como criterios de exclusión al personal de ventas y soporte técnico; se utilizó la técnica de la encuesta, y como instrumento se empleó el cuestionario (con ayuda de la herramienta Google Forms), a través del cual se procedió a recopilar los datos necesarios de forma anónima y de esta manera obtener información del personal de CETI.

El instrumento estuvo constituido por 11 reactivos los cuales tuvieron por finalidad conocer la experiencia del usuario en la utilización e implementación de plataformas e-learning para el desarrollo de cursos a distancia. La encuesta fue aplicada de manera virtual a la población en estudio; con la aceptación y respuesta de ésta se procedió al procesamiento de la información haciendo uso de la Hoja de Cálculo de Microsoft Excel. Luego de trasladar los resultados en tablas, estos fueron graficados e interpretados para el entendimiento del problema; posteriormente los resultados encontrados sirvieron como insumo para la elaboración del modelo propuesto.

\section{Resultados}

Los resultados de la investigación muestran que, en la mayoría de los docentes y administrativos del CETI, un gran porcentaje (70\%) hace uso de alguna plataforma e-learning. A pesar de ello, pocos han realizado la implementación de una de estas (5\%), y menor cantidad de encuestados manifiestan haber utilizado el estándar ISO/IEC 25000 para evaluar o desarrollar algún producto de software.

Por otro lado, en relación con su experiencia utilizando alguna plataforma e-learning, se evidencia que tienen algún grado de conformidad con respecto al desempeño de estas; sin embargo, cabe recalcar que en plena era digital muchos encuestados $(52,6 \%)$ no hacen uso de su 
dispositivo móvil (llámese smartphone o tablet) para acceder a una plataforma e-learning, según se indica en la tabla 1.

Tabla 1. Uso de la plataforma e-learning en dispositivos móviles

\begin{tabular}{lcc}
\hline Escala & Frecuencia (fi) & Porcentaje (\%) \\
\hline Siempre & 3 & $15.8 \%$ \\
Casi siempre & 4 & $21.1 \%$ \\
Casi nunca & 2 & $10.5 \%$ \\
Nunca & 10 & $52.6 \%$ \\
& 19 & $100.0 \%$ \\
& Total & Fuente: Elaboración propia
\end{tabular}

En esa misma línea, se deseaba conocer a partir de la experiencia del encuestado cuál de las plataformas le brinda la sensación de ser más completa en cuanto a la escalabilidad, aspecto amigable - intuitivo, funcional; y se evidenció que el 25\% optaba por la plataforma Blackboard y Moodle respectivamente; luego Canvas LMS con 20\%; después Chamilo con 10\%; y por último están GoToWebinar y Platzi con un 5\% cada una, tal como se muestra en la figura 1.

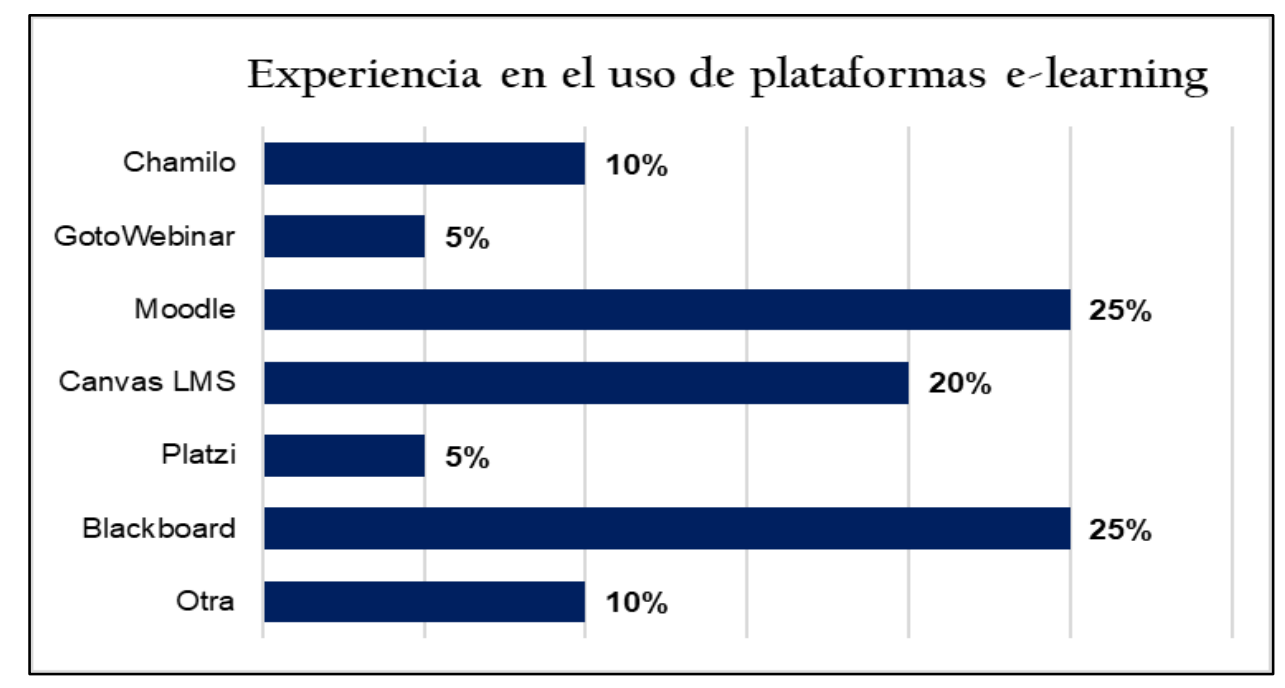

Figura 1. Experiencia en el uso de plataformas e-learning Fuente: Elaboración propia 
REVISTA DE LA UNIVERSIDAD DEL ZULIA. 3e época. Año 12 N 32, 2021 Eder Nicanor Figueroa Piscoya et al. // Modelo de calidad para plataformas e-learning ... 330-343

Finalmente, era necesario llevar al encuestado a un escenario donde pudiera adentrarse en el proceso de implementación de una plataforma e-learning; para ello se le invitó a elegir ciertas características que contendría una plataforma. El 80\% de los encuestados señalaron que el seguimiento de los avances del estudiante en tiempo real es la característica más notable que incorporarían en la implementación de una plataforma e-learning. Por su parte, un 60\% precisa que la evaluación de los estudiantes en períodos de tiempo semanal o quincenal es vital; y otra característica es que debería permitirle al docente la creación de juegos por cada tema del curso, con el $55 \%$, según se evidencia en la figura 2.

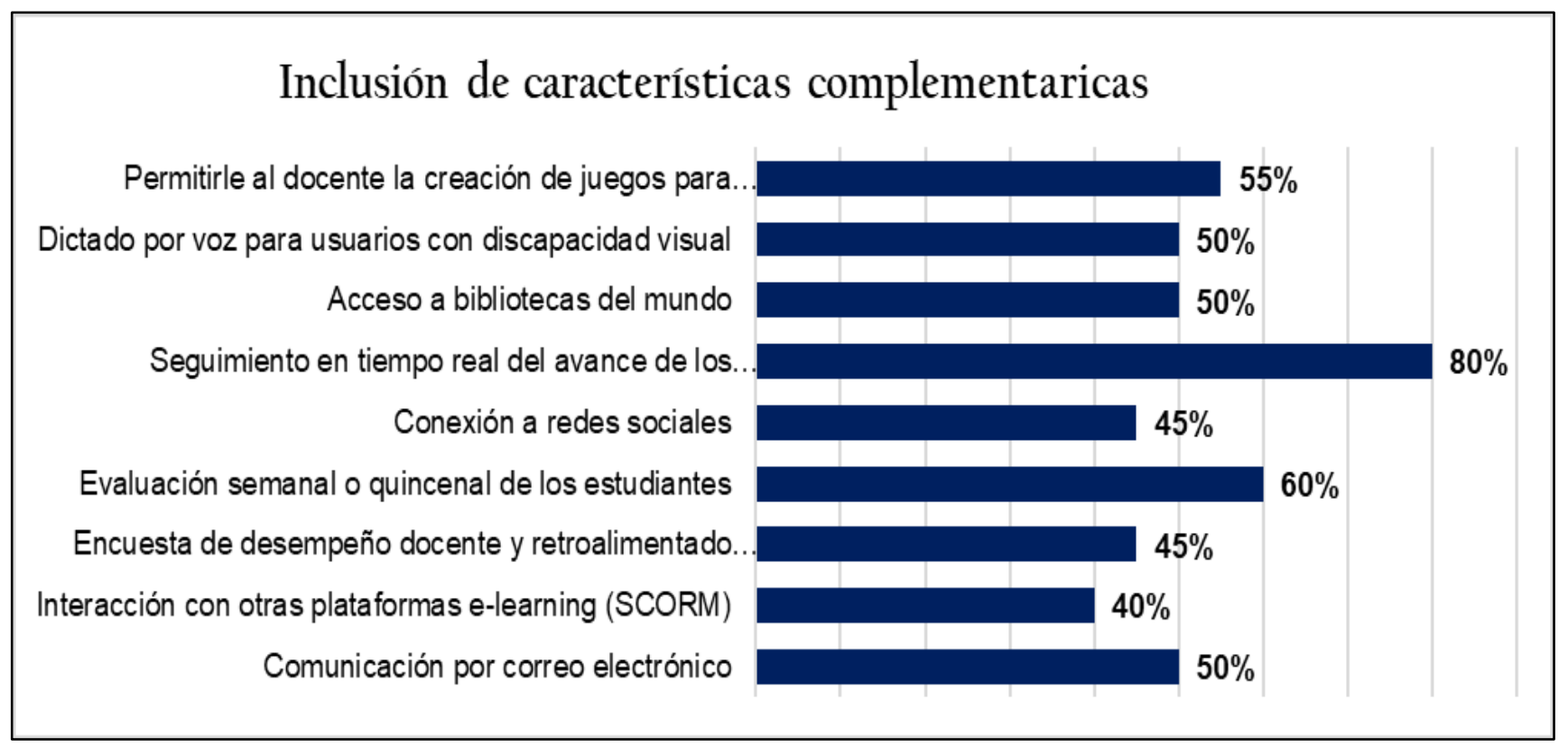

Figura 2. Inclusión de principales características complementarias si el encuestado participaría en el proceso de construción de una plataforma e-learning Fuente: Elaboración propia

Debido a todo lo recopilado en el desarrollo de la presente investigación se procedió a elaborar y proponer un modelo, el cual se resume en la figura 3.

Los atributos serán los pilares del modelo, posicionándose en la base de este, a fin de indicar las propiedades que las plataformas e-Learning deben tener para dar satisfacción al ser utilizadas por los docentes, administrativos y estudiantes. La inobservancia de estos atributos conduciría a la pérdida total de calidad. En el modelo propuesto se han incluido nueve atributos 
REVISTA DE LA UNIVERSIDAD DEL ZULIA. 3época. Año 12 N 32, 2021 Eder Nicanor Figueroa Piscoya et al. // Modelo de calidad para plataformas e-learning ... 330-343

DOI: http://dx.doi.org/10.46925//rdluz.32.20

para plataformas e-Learning, obtenidos sobre las bases de las contribuciones de (Castro et al., 2013).

El modelo toma robustez al incorporar la norma internacional ISO/IEC 25000, la cual refuerza los nueve atributos de las plataformas e-Learning mencionados anteriormente. Se debe precisar que las características y subcaracterísticas fueron escogidas más precisamente de la ISO/IEC 25010 e ISO/IEC 25012, ambos incluidos en el estándar, que hacen referencia al modelo de calidad en los productos de soft ware y al modelo de calidad de los datos respectivamente (ISO 25000, 2020). Cada una de las subcaracterísticas incluye un conjunto de métricas o aspectos básicos debidamente seleccionados y detallados, que las plataformas e-Learning deben tener para favorecer su calidad final.

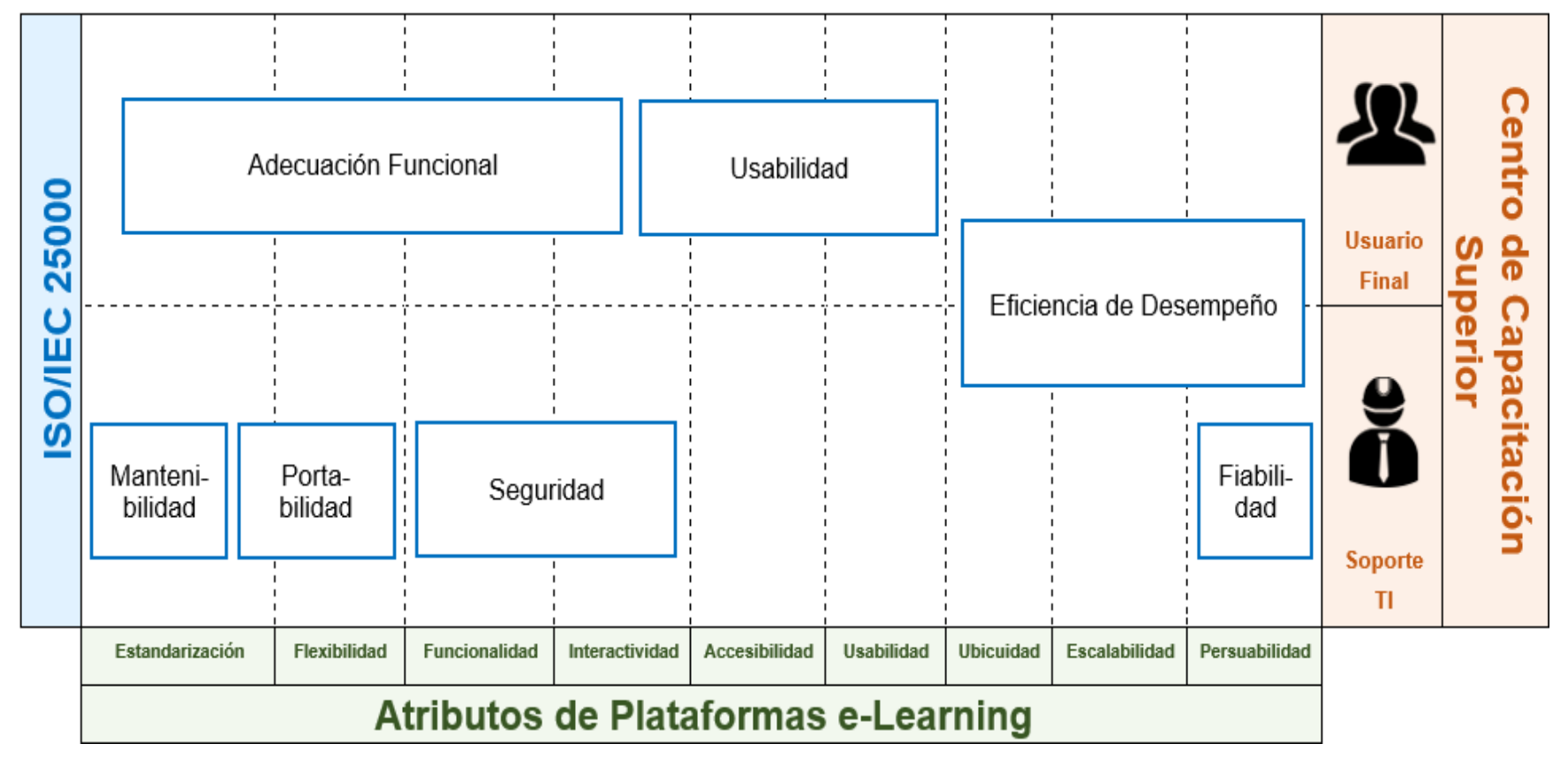

Figura 3. Modelo de calidad para plataformas e-learning en centros de entrenamiento superior basado en normas ISO/IEC 25000.

Fuente. Elaboración propia

Por último, el modelo propuesto involucra a los actores, quienes al concebir la idea de adquirir una plataforma e-learning en el centro de entrenamiento superior, por lo general, tendrán dos opciones a seguir: inclinarse por elegir plataformas LMS ya desarrolladas (libres o comerciales); o decidir construir una plataforma e-Learning personalizada. En el primer caso el 
REVISTA DE LA UNIVERSIDAD DEL ZULIA. 3e época. Año 12 N³2, 2021

Eder Nicanor Figueroa Piscoya et al. // Modelo de calidad para plataformas e-learning ... 330-343

DOI: http://dx.doi.org/10.46925//rdluz.32.20

modelo propuesto se utilizará para evaluar los aspectos de calidad siguiendo las métricas adaptadas para los productos de software LMS libres o comerciales disponibles en el mercado (Viñas, 2017). En el segundo caso, el modelo propuesto se utilizará como la pauta que debe tener el equipo durante todo el proceso de desarrollo del software (recopilando los requerimientos de los stakeholders, diseñar la arquitectura y construir las bases de datos, etc.) para lograr construir una plataforma e-learning de calidad, ofreciendo un alto grado de satisfacción al adecuarse a todas sus necesidades específicas (Rodríguez, 2020).

\section{Discusión}

El modelo está nutrido por una exploración bibliográfica de la familia de normas ISO/IEC 25000, artículos e investigaciones como la de Vaca \& Jácome (2018) y Baldeón (2015), quienes concuerdan con la idea que esta investigación pretende reforzar, que para satisfacer al personal que utiliza el producto software, este último debe alcanzar un alto grado de calidad y que para lograrlo es de gran ayuda la utilización de estándares ISO.

Por otro lado, una de los artículos que llamó la atención de los investigadores al realizar la revisión bibliográfica fue la de Cocunubo-Suarez et al. (2018), quienes llegan a concluir que para realizar una evaluación a alguna plataforma e-Learning (o Entornos Virtuales de Enseñanza Aprendizaje - EVEA) "es fundamental tener en cuenta 4 aspectos pedagógicos (contenido, modelo, evaluación, interfaz de usuario, herramientas); sin relación con ninguna característica del estándar ISO 25000" (p. 144); y es aquí donde entramos a discusión y llegamos a argumentar que dos de los aspectos pedagógicos que se mencionan (herramientas e interfaz de usuario), al no relacionarlos con ninguna característica del estándar ISO mencionado, no se tomarían en cuenta durante el proceso de medición de la calidad por medio del estándar ISO/IEC 25000, y esto ocasionaría que la calidad aportada a la plataforma e-Learning sea deficiente. Debido a ello, el presente artículo incluirá estos aspectos pedagógicos dentro del modelo propuesto para obtener una correspondencia entre los temas ligados a la enseñanza y la calidad brindada por las características del estándar ISO/IEC 25000 que darán soportes a esos aspectos pedagógicos.

Otra de las observaciones que los investigadores se percataron al momento de realizar este estudio es la poca intervención e inclusión explícita de personas especialistas (actores) 
dentro de los modelos de evaluación de la calidad del producto software, específicamente de las plataformas e-Learning. Siempre que se menciona algún actor es tomado como una persona externa a los modelos y solamente interviene en etapas tardías del proceso de desarrollo o en auditorías de calidad del producto. La acotación que hace (Baldeón, 2015) en su investigación, nos indica que "la aplicación del método para la evaluación de calidad permitió asegurar que el equipo de desarrollo plasme adecuadamente lo que el usuario necesita". Esto da un claro aporte al conseguir una relación entre equipo de desarrollo, utilización del método de evaluación y usuarios finales. En este artículo el modelo propuesto tiene muy en cuenta a las personas que deben tomar parte en todo momento del proceso de evaluación, tales como personal especializado y usuario final, de tal modo que trabajando conjuntamente puedan llegar a adquirir una plataforma e-Learning que cubra todos (o casi todos) los requerimientos, y así la calidad del software que ellos merecen esté garantizada.

Por último, se debe destacar que el modelo hace énfasis en aportar calidad suficiente a la plataforma e-Learning para que al ser utilizada por el docente le ayude en el proceso de enseñanza-aprendizaje; tanto en una etapa pasiva del proceso, al elaborar y construir su material bajo una estrategia didáctica; así como también en la etapa activa del proceso, donde habrá participación del estudiante con quien desarrollará el material, evaluará y realizará el respectivo seguimiento (Meneses, 2007). Es debido a todo este proceso que el modelo propuesto basado en normas ISO/IEC 25000 será de gran ayuda para que los centros de entrenamiento superior logren obtener la plataforma e-Learning adecuada.

\section{Conclusiones}

Por los resultados obtenidos, en los centros de entrenamiento superior se puede medir y mejorar la calidad de una plataforma e-Learning, cumpliendo para ello con nueve atributos: estandarización, flexibilidad, funcionalidad, interactividad, accesibilidad, usabilidad, ubicuidad, escalabilidad, persuabilidad. Y para lograr este cometido, cada atributo debe estar soportado por una o más características seleccionadas de la familiar de normas ISO/IEC 25000, cada una con sus métricas o aspectos básicos. 
REVISTA DE LA UNIVERSIDAD DEL ZULIA. 3época. Año $12 \mathrm{~N}^{\circ}$ 32, 2021

Eder Nicanor Figueroa Piscoya et al. // Modelo de calidad para plataformas e-learning ... 330-343

DOI: http://dx.doi.org/10.46925//rdluz.32.20

El análisis hecho en la documentación muestra que los modelos de evaluación de calidad de software no precisan a los actores que deben involucrarse en la medición. Por consiguiente, el modelo presentado en este artículo propone incluir al menos dos actores que form an parte del centro de entrenamiento superior, quienes estarán inmersos en el proceso de medición y evaluación de la calidad en las plataformas e-Learning.

Se espera que el modelo basado en la familia de estándares internacionales ISO/IEC 25000 propuesto en este artículo sirva como herramienta fundamental dentro de los centros de entrenamiento superior que deseen determinar la calidad en sus plataformas e-Learning, y de esta manera lograr que la tecnología ayude a sus docentes en el proceso de enseñanzaaprendizaje. Además, el modelo es presentado para que los investigadores interesados ahonden en los temas relacionados con la construcción y evaluación de plataformas e-Learning de calidad siguiendo normas internacionales ISO.

\section{Referencias}

Baldeón, E. J. (2015). Método para la evaluación de la calidad del sotfware basado en ISO/IEC [Tesis de Maestría]. In Universidad de San Martín de Porres. http://repositorio.usmp.edu.pe/bitstream/handle/20.500.12727/1480/baldeon_vej.pdf?sequence= l\&:isAllowed $=y$

Bernal, C. A. (2010). Metodología de la Investigación. In Pearson Educación (Tercera). Pearson Educacion.

Blázquez Barba, M., Gómez Romero, D., Frontaura Fernández, I., Camacho Ojeda, A., Rodríguez Salas, F. M., \& Toriz Cano, H. (2018). Uso de Internet por los adolescentes en la búsqueda de información sanitaria. Atención Primaria, 50(9), 547-552. https://doi.org/10.1016/j.aprim.2017.06.005

Callejas-Cuervo, M., Alarcón-Aldana, A. C., \& Álvarez-Carreño, A. M. (2017). Modelos de calidad del software, un estado del arte. ENTRAMADO, 13(1), 236-250. https://doi.org/10.1804l/entramado.2017vl3nl.25125

Castro, S. M., Clarenc, C. A., Lopez de Lenz, C., Moreno, M. E., \& Tosco, N. B. (2013). Analizamos 19 plataformas colaborativas de e-learning: Investigación colaborativa sobre LMS. In Congreso Virtual Mundial de e-Learning. https:/docplayer.es/485121-Analizamos-19plataformas-de-e-learning-investigacion-colaborativa-sobre-lms.html 
REVISTA DE LA UNIVERSIDAD DEL ZULIA. 3aépoca. Año $12 \mathrm{~N}^{\circ}$ 32, 2021 Eder Nicanor Figueroa Piscoya et al. // Modelo de calidad para plataformas e-learning ... 330-343

DOI: http://dx.doi.org/10.46925//rdluz.32.20

Cocunubo-Suarez, J. I., Parra-valencia, J. A., \& Otárola-Luna, J. E. (2018). Propuesta para la evaluación de Entornos Virtuales de Enseñanza Aprendizaje con base en estándares de Usabilidad. 21(41), 135-147. https://dialnet.unirioja.es/servlet/articulo?codigo=6289039

Deming, W. E. (1989). Calidad, productividad y competitividad: la salida de la crisis. In Google Libros

(Cambridge). https://books.google.com.pe/books?id=d9WL4BMVHi8C\&printsec=frontcover $\# \mathrm{v}=$ onepage\& $\& q$ $\& \mathrm{ff}=\mathrm{false}$

Gómez, D. A. (2015). Analítica Visual en Elearning [Tesis de Doctorado]. In Universidad de Salamanca. https://gredos.usal.es/handle/10366/125950

ISO 25000. (2020). La familia de normas ISO/IEC 25000. ISO 25000. https://iso25000.com/

James, J. (20018). CHAOS Report: Decision Latency Theory: It Is All About the Interval. In The Stadish

Group.

https://books.google.com.pe/books?id=WVlQDwAAQBAJ\&printsec=frontcover\&hl=es\&sourc $\mathrm{e}=\mathrm{gbs}$ ge_summary_r\&sad=0 $\# \mathrm{v}=$ onepage $\& \mathrm{q} \& \mathrm{Qf}=$ false

Meneses, G. (2007). El proceso de enseñanza- aprendizaje: el acto didáctico. In NTIC, Interacción y Aprendizaje en la Universidad (pp. 31-65). https:/www.tdx.cat/bitstream/handle/10803/8929/Elprocesodeensenanza.pdf

Miguel, P, Mauricio, D., \& Rodríguez, G. (2014). A Review of Software Quality Models for the Evaluation of Software Products. International Journal of Software Engineering \& Applications, 5(6), 31-53. https://doi.org/10.5121/ijsea.2014.5603

Morales Ramírez, A., Zacatenco Cruz, J. D., Luna Luna, M., García Lozano, R. Z., \& Hidalgo Cortés, C. (2020). Acceso y actitud del uso de Internet entre jóvenes de educación universitaria. Revista Digital de Investigación En Docencia Universitaria, 14(1), 20-29. https://doi.org/10.19083/ridu.2020.1174

PMI. (2017). Success Rates Rise: Transforming the high cost of low performance. Pulse of the Profession - 9th Global Project Management Survey, 1-32. https:/www.pmi.org//media/pmi/documents/public/pdf/learning/thought-leadership/pulse/pulse-of-the-profession2017.pdf

Ramos, R. C. B., Villagran, N. V., Yoo, S. G., \& Quina, G. N. (2018). Software Quality Assessment Applied for the Governmental Organizations using ISO/IEC 25000. 2018 5th International Conference on EDemocracy and EGovernment, ICEDEG 2018, 311-316. https://doi.org/10.1109/ICEDEG.2018.8372327

Rodríguez, M. (2020). Desarrollo de Software a la Medida VS Software Comercial. Medium. https://medium.com/@MiltonRod/desarrollo-de-software-a-la-medida-vs-software-comercial7c3329f876fe 
REVISTA DE LA UNIVERSIDAD DEL ZULIA. 3época. Año $12 \mathrm{~N}^{\circ}$ 32, 2021 Eder Nicanor Figueroa Piscoya et al. // Modelo de calidad para plataformas e-learning ... 330-343 DOI: http://dx.doi.org/10.46925//rdluz.32.20

Sanchez, W. (2011). La usabilidad en Ingeniería de Software : definición y características. IngNovación, 02, 7-2l. https://core.ac.uk/download/pdf/4726496l.pdf

Vaca, T., \& Jácome, A. (2018). Calidad de software del módulo de talento humano del sistema informático de la Universidad Técnica del Norte bajo la norma ISO/IEC 25000. RerearchGate, May, ResearchGate. https://www.researchgate.net/publication/325022337_Calidad_de_software_del_modulo_de_tal ento_humano_del_sistema_informatico_de_la_Universidad_Tecnica_del_Norte_bajo_la_norma_I SOIEC_25000

Viñas, M. (2017). La importancia del uso de plataformas educativas. Letras, 1(6), 157-169. http://sedici.unlp.edu.ar/handle/10915/61390 